\title{
Proceedings
}

\section{1st International Workshop on Safe Control of Connected and Autonomous Vehicles}

\section{SCAV 2017}

21 April 2017

Pittsburgh, Pennsylvania, USA

(This workshop is part of CPS Week, 18-21 April 2017) 
The Association for Computing Machinery

2 Penn Plaza, Suite 701

New York New York 10121-0701

ACM COPYRIGHT NOTICE. Copyright $\odot 2017$ by the Association for Computing Machinery, Inc. Permission to make digital or hard copies of part or all of this work for personal or classroom use is granted without fee provided that copies are not made or distributed for profit or commercial advantage and that copies bear this notice and the full citation on the first page. Copyrights for components of this work owned by others than ACM must be honored. Abstracting with credit is permitted. To copy otherwise, to republish, to post on servers, or to redistribute to lists, requires prior specific permission and/or a fee. Request permissions from Publications Dept., ACM, Inc., fax + 1 (212) 869-0481, or permissions@acm.org.

For other copying of articles that carry a code at the bottom of the first or last page, copying is permitted provided that the per-copy fee indicated in the code is paid through the Copyright Clearance Center, 222 Rosewood Drive, Danvers, MA 01923,

$$
+1-978-750-8400,+1-978-750-4470 \text { (fax). }
$$

\section{Notice to Past Authors of ACM-Published Articles}

ACM intends to create a complete electronic archive of all articles and/or other material previously published by ACM. If you have written a work that was previously published by ACM in any journal or conference proceedings prior to 1978, or any SIG Newsletter at any time, and you do NOT want this work to appear in the ACM Digital Library, please inform permissions@acm.org, stating the title of the work, the author(s), and where and when published.

ACM ISBN: 978-1-4503-4976-5

Editorial production by Randall Bilof Cover art production by Mark Bartosik

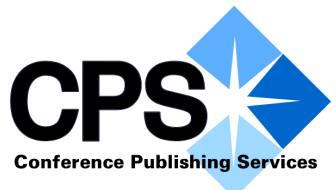

IEEE Computer Society Conference Publishing Services (CPS)

http://www.computer.org/cps 


\section{1st International Workshop on Safe Control of Connected and Autonomous Vehicles}

\section{SCAV 2017}

\section{Table of Contents}

Message from the Program Chairs

..vii

Conference Organization

\section{Keynote}

Challenges in Autonomous Vehicle Validation 3 Philip Koopman

\section{Invited Paper}

Controlling for Unsafe Events in Dense Traffic through Autonomous Vehicles

Daniel B. Work, Raphael Stern, Fangyu Wu, Miles Churchill, Shumo Cui,

Hannah Pohlmann, Benjamin Seibold, Benedetto Piccoli, R'mani Bhadani,

Matt Bunting, Jonathan Sprinkle, Maria Laura Delle Monache,

Nathaniel Hamilton, and R'mani Haulcy

\section{Technical Papers}

Osiris: A Tool for Abstraction and Verification of Control Software with Lookup Tables

Nikos Arechiga, Sumanth Dathathri, Shashank Vernekar, Nagesh Kathare,

Sicun Gao, and Shinichi Shiraishi

Using ID-Hopping to Defend Against Targeted DoS on CAN

Abdulmalik Humayed and Bo Luo

A Game-Theoretic Approach to Secure Control of Communication-Based

Train Control Systems Under Jamming Attacks.

Zhiheng Xu and Quanyan Zhu

A Game-Theoretic Approach and Evaluation of Adversarial Vehicular Platooning Imran Sajjad, Rajnikant Sharma, and Ryan Gerdes 
Vehicle Platooning Simulations with Functional Reactive Programming... Bernd Finkbeiner, Felix Klein, Ruzica Piskac, and Mark Santolucito

Author Index.. 


\section{Message from the SCAV 2017 Program Chairs}

Welcome to the First International Workshop on Safe Control of Connected and Autonomous Vehicles (SCAV 2017)! We are proud to be able to conduct this workshop at CPS Week 2017.

Theme and Goals: Among the many challenges of making systems acceptably safe, dependable, resilient, and secure, we want to investigate the relationships of two complementary views:

- Safety by designing safe system dynamics (aka "safety of the intended function"): Engineers take care of implementing function enhancements (e.g., collision avoidance, safety braking) that are responsible for mitigating the hazardous situations typical of an application.

- Safety by improving resilience, dependability, and security: When performing, system functions can exhibit various anomalies (e.g., due to faults, intrusions, attacks, misuse), thus violating the intended function. Engineers take care of avoiding, detecting, and containing such anomalies by improving various properties of a system design.

Our objective is to discuss novel challenges and approaches within and relationships between these two views. Employing mathematical models, formal methods, and analysis tools and also seeking exchanges with practitioners help us to identify the core challenges and implications of higher levels of automation on the design and the engineering of safety concepts for automated and autonomous, individual and connected vehicles and traffic.

Agenda: With the help of the 23 members of our program committee and external reviewers and directly based on 17 peer reviews, we accepted 5 out of the 6 submissions for this single-day workshop. We are happy to enhance our paper session in the morning with several invited talks, altogether stimulating group discussions in the afternoon. The workshop closes with the presentation and summary of important findings from the discussions, culminating in a closing panel. We plan to publish our findings in an extra short paper.

Acknowledgments: Finally, we are very grateful to everyone who is involved in the organization and conduct of this workshop, the program committee, external reviewers, submitting authors, invited speakers, CPS Week organizers, and our home faculties.

Mario Gleirscher, Technical University of Munich

Stefan Kugele, Technical University of Munich

Jonathan Sprinkle, University of Arizona

SCAV 2017 Program Chairs 


\section{SCAV 2017 Conference Organization}

\section{Program Chairs}

Mario Gleirscher, Technical University of Munich, DE Stefan Kugele, Technical University of Munich, DE Jonathan Sprinkle, University of Arizona, USA

\section{Program Committee}

Matthias Althoff, Technical University of Munich, DE Ezio Bartocci, Technical University Vienna, AT

Karl Berntorp, Mitsubishi Electric Research Labs, USA

Manfred Broy, Technical University of Munich, DE Herman Bruyninckx, University Leuven, BE

Tomas Bures, University Prague, CZ Wolfram Burgard, University Freiburg, DE Simon Burton, Bosch, DE

Nicolas D'Ippolito, University of Buenos Aires, AR Antonio Filieri, Imperial College London, UK Ilias Gerostathopoulos, Technical University of Munich, DE Jeremie Guiochet, LAAS, FR

Mats Heimdahl, University of Minnesota, USA Alessia Knauss, Chalmers UT, SE

Alois Knoll, Technical University of Munich, DE Martin Leucker, University Lübeck, DE Stefan Leue, University Konstanz, DE Alessandro Papadopoulos, Mälardalen University, SE Benedetto Piccoli, Rutgers University, USA Rosario Pugliese, University Firenze, IT Benjamin Seibold, Temple University, USA Mike Wagner, CMU, USA Dan Work, UIUC, USA

\section{External Reviewer}

Klaus Becker, fortiss GmbH, DE 